\title{
FOLFOX-4 Regimen as a First-line Therapy for Cuban Patients with Metastatic Colorectal Cancer
}

\author{
Leonardo Lami, MD, Fernando Areces, MD, Juan J Lence, MD, María A Arbesú, MS
}

\begin{abstract}
Introduction In Cuba, colorectal cancer (CRC) is the malignant neoplasm with the fourth-highest incidence and third-highest mortality. Over one-third of CRC patients exhibit metastatic disease at the time of diagnosis. Standard treatment for metastatic CRC is a 5-fluorouracil (5-FU) + Folinic Acid (FA) continuous infusion regimen. International studies have shown, however, that systemic therapy using oxaliplatin combined with 5-FU and FA (FOLFOX-4) improves results in terms of both tumor response and survival in patients with inoperable metastatic CRC.
\end{abstract}

Objective Evaluate the FOLFOX-4 regimen as a first-line therapy for patients with inoperable metastatic CRC in Cuba.

Methods FOLFOX-4 therapy was administered to 56 patients with metastatic CRC, in a treatment cycle repeated every 2 weeks for $6-8$ cycles. Patients were followed up for a period of 2 years.

\section{INTRODUCTION}

Colorectal cancer (CRC) currently constitutes a major epidemiological problem due to high rates of frequency and mortality; in Europe and North America, it is the third most frequent type of malignant tumor and the second leading cause of cancer death. Worldwide, an estimated 1 million new cases and half a million deaths are attributed to CRC annually.[1] When first diagnosed, $>30 \%$ of CRC patients present metastatic disease, and $50-60 \%$ eventually develop metastasis or advanced disease. The prognosis for these patients is devastating, with a 5-year survival rate $\leq 5 \%$.[2] In Cuba, CRC is the malignant neoplasm with the fourthhighest incidence and third-highest mortality.[3]

In the last 40 years, CRC has transitioned from an incurable chemo-resistant tumor to an incurable chemo-sensitive tumor with a more favorable evolution. Numerous studies carried out in the last two decades confirm that systemic chemotherapy extends the period of progression-free disease and increases survival in patients treated for metastatic CRC. For more than 30 years, 5-fluorouracil (5-FU) has been the first-line agent in this fight, included in the majority of palliative chemotherapy regimens. Its efficacy has improved over time through biomodulation with folinic acid and the use of continuous infusion schedules, but these modifications have not made a significant impact on survival.[4]

The emergence of new anticancer drugs in the 1990s opened a door in the search for combinations that would increase the tumor response rate with a tolerable toxicity pattern, thereby making a positive impact on patient survival.[4,5] Among these drugs, oxaliplatin (a third-generation platinum derivative) stands out. In multiple international meta-analyses, and through its use in various treatment regimens, it has proved to be a safe alter-
Results Objective response was attained in $44.6 \%$ of patients, and complete response in $12.5 \%$. Median duration of response and of progression-free survival was 9.6 and 8.9 months, respectively. Estimated survival at 2 years was $17 \%(95 \% \mathrm{Cl}: 6.89$ 26.8). The most frequent adverse events were nausea, vomiting, diarrhea and neutropenia, the majority grade $1-2$, according to Common Terminology Criteria for Adverse Events (CTCAE) classification.

Conclusions In patients studied, the FOLFOX-4 combination was shown to be an effective and well-tolerated therapeutic option for treating inoperable metastatic colorectal cancer.

Keywords: FOLFOX, antineoplastic combined chemotherapy protocols, anticancer drug combinations, antineoplastic agents, polychemotherapy, colorectal neoplasms, colorectal cancer, neoplasm metastasis, neoplasm invasiveness native, demonstrating better results than the 5-FU + folinic acid (FA) continuous infusion regimen in terms of tumor response and survival.[6]

In Cuba, the 5-FU + FA combination has been standard treatment for metastatic CRC. However, a study carried out at the National Oncology and Radiobiology Institute in Havana (INOR, its Spanish acronym) analyzing survival in 39 patients with disseminated CRC treated at INOR between 1974 and 1980 with either supportive care or a 5-FU + FA chemotherapy regimen, reported a $0 \%$ 11-month survival rate.[7] For this reason, incorporation of new treatment regimens that can effectively increase survival is imperative.

The objective of this study is to describe results of treatment using the FOLFOX-4 combination as first-line therapy in patients with inoperable metastatic CRC treated at INOR.

\section{METHODS}

\section{Participants}

Inclusion criteria This study included 56 patients aged $>18$ years, diagnosed with inoperable metastatic CRC and admitted to INOR between January 1, 2001 and December 31, 2004. None of the patients had received chemotherapy for their metastatic disease, although they may have received surgery or prior chemotherapy with 5-FU at the time of initial diagnosis. All had a confirmed histological diagnosis of colorectal adenocarcinoma with at least one metastatic lesion $\geq 10 \mathrm{~mm}$ located in the lung or the skin, or $\geq 20 \mathrm{~mm}$ located in the liver, bones or soft tissues; performance status $0-2$ on the World Health Organization (WHO) scale[8]; and life expectancy $>3$ months.

Exclusion criteria Not included were patients with a history of malignant neoplasm or metastasis in the central nervous system 
(CNS), neurological or psychiatric disorders, decompensated cardiovascular disease, malabsorption syndrome, surgical removal of any part of the upper digestive tract, or patients who were pregnant or lactating.

Ethical considerations Patients received information about the risks and benefits of the proposed treatment and gave written informed consent to participate in the study. The study was approved by the INOR Ethics Committee.

\section{Treatment Design}

FOLFOX 4 Regimen Patients received the following treatment:

- Oxaliplatin (Eloxatin, Sanofi-Synthelabo, France), $85 \mathrm{mg} / \mathrm{m}^{2}$, by 2-hour intravenous infusion, day 1 of each cycle.

- Folinic Acid (Dalisol, Lemery, Mexico), $200 \mathrm{mg} / \mathrm{m}^{2}$ by 2-hour infusion, days 1 and 2 of each cycle.

- $\quad 5-F U$ (Flurox, Lemery, Mexico), $400 \mathrm{mg} / \mathrm{m}^{2}$ by intravenous bolus $+600 \mathrm{mg} / \mathrm{m}^{2}$ by 22-hour intravenous infusion, days 1 and 2 of each cycle.

FA and oxaliplatin infusions were administered simultaneously on day 1 , followed by administration of 5-FU. Patients remained hospitalized at INOR while receiving treatment.

Cycles were repeated every 2 weeks, and 6-8 cycles were planned. Patients exhibiting tumor response to treatment or stable disease could continue with more cycles if indicated by clinical criteria.

Response to treatment was evaluated according to the objective response rate, duration of the objective response obtained, progression-free survival, and overall survival. The treatment's toxicity profile was also evaluated. Patients were followed-up for at least 2 years after termination of treatment.

Objective response to treatment was evaluated using $\mathrm{Re}$ sponse Evaluation Criteria in Solid Tumors (RECIST) guidelines. [9] Clinical evaluation was performed monthly during treatment, once during the 4-week period following termination of treatment, and every 3 months thereafter. Imaging studies-chest X-rays, abdominal ultrasound and computerized axial tomography (CAT) of the metastasis site-were also made at the halfway point and at termination of the planned treatment, and when indicated by the treating physician due to symptoms of progression. Objective response rate $(R R)$ was calculated as the total number of patients attaining complete or partial response at termination of treatment, expressed as a percentage of the total number of patients receiving treatment.

Median duration of response to treatment was calculated from the treatment termination date until the date on which signs of disease progression appeared in those patients who achieved complete or partial response.

Progression-free survival (PFS) was calculated as the time elapsed between initiation of treatment and appearance of signs of disease progression after response was attained, or signs of progressive disease, or death from any cause, or the last known date of progression-free disease. Evaluation of progression-free survival and overall survival was made every 3 months following termination of treatment.
Overall survival (OS) was calculated as the time elapsed from initiation of treatment until the patient's death from any cause, or until the last entry in the patient's medical chart at the time results were analyzed.

Safety profile was evaluated in all patients who received at least one dose of chemotherapy. Toxicity was evaluated at least monthly until 4 weeks after termination of treatment and every 3 months thereafter. This evaluation included laboratory parameters (complete hemogram, renal and hepatic profiles), clinical assessment of adverse reactions, and classification of adverse events according to the Common Terminology Criteria for Adverse Events (CTCAE): grade 1=mild adverse event; grade $2=$ moderate adverse event; grade 3=severe adverse event; and grade 4= life-threatening adverse event.[10] Relative frequency of adverse events was expressed as a percentage.

In cases of severe toxicity, (grade 3-4 supported by clinical criteria), oxaliplatin and 5 -FU doses were both reduced by $25 \%$ after the first event and by $50 \%$ after the second event.

Chemotherapy was discontinued in cases of documented disease progression or limiting toxicity. Administration of 5-FU was continued in patients for whom oxaliplatin administration was suspended due to toxicity.

Statistical analysis Overall survival and progression-free survival rates were calculated using the Kaplan-Meier method $(95 \%$ $\mathrm{Cl}$ ). The chi-square test for independence was used to determine correlation between qualitative variables $(p<0.05)$. The prognostic value of some factors was demonstrated by comparing survival curves for those subgroups using the log-rank test $(p<0.05)$. Data was processed with the Stata statistical package, version 8 for Windows and SPSS version 11.5. Results were represented in tables and graphs.

\section{RESULTS}

Clinical-epidemiological profile Mean age of patients was 56 years, and $73 \%$ had a $0-1$ performance status. The colon was the most frequent primary tumor site ( $70 \%$ of patients), and the liver was the organ most frequently affected by metastatic disease $(66 \%)$. About $40 \%$ of patients had received neo-adjuvant or adjuvant chemotherapy with 5-FU before diagnosed with disseminated or metastatic disease (Table 1).

Treatment application All 56 patients received at least 2 treatment cycles; $94.6 \%$ (53) received a minimum of 4 cycles; $80.4 \%$ (45) received 6 or more cycles, for an average of 5.8 cycles per patient. Doses were reduced in 16 patients $(28.6 \%)$, primarily due to grade-3 adverse reactions. The therapeutic regimen was interrupted in 15 patients $(26.8 \%)$; the most common reasons were severe toxicity and disease progression.

Treatment efficacy According to RECIST criteria, 7 patients $(12.5 \%)$ attained complete response (CR), and 18 patients $(32.1 \%)$ partial response (PR), for a response rate (RR) of $44.6 \%$. Median response duration was 9.6 months (Table 2).

The response rate was slightly higher (but not statistically significant) in women than in men (50\% and $35 \%$, respectively) $(p<0.78)$, and in patients with primary tumor site in the colon 
Table 1: Clinical-Epidemiological Characteristics of Patients with Inoperable Metastatic Colorectal Cancer Treated with FOLFOX-4, INOR, Havana (2001 to 2004)

\begin{tabular}{|c|c|c|}
\hline \multirow{2}{*}{ Characteristics } & \multicolumn{2}{|c|}{ Patients } \\
\hline & No. & $\%$ \\
\hline $\begin{array}{l}\text { Sex } \\
\text { Male } \\
\text { Female }\end{array}$ & $\begin{array}{l}30 \\
26\end{array}$ & $\begin{array}{l}53.6 \\
46.4\end{array}$ \\
\hline $\begin{array}{l}\text { Age (years) } \\
\text { Mean } \\
\text { Range }\end{array}$ & & \\
\hline $\begin{array}{l}\text { Performance Status (WHO) } \\
0 \\
1 \\
2\end{array}$ & $\begin{array}{r}1 \\
40 \\
15\end{array}$ & $\begin{array}{r}1.8 \\
71.4 \\
26.8\end{array}$ \\
\hline $\begin{array}{l}\text { Primary Tumor Site } \\
\text { Colon } \\
\text { Rectum }\end{array}$ & $\begin{array}{l}39 \\
17\end{array}$ & $\begin{array}{l}69.6 \\
30.4\end{array}$ \\
\hline $\begin{array}{l}\text { Previous Therapy } \\
\text { Surgery } \\
\text { Chemotherapy with 5-FU }\end{array}$ & $\begin{array}{l}34 \\
22\end{array}$ & $\begin{array}{l}60.7 \\
39.3\end{array}$ \\
\hline $\begin{array}{l}\text { Metastatic Location } \\
\text { Liver } \\
\text { Soft Tissues } \\
\text { Lung } \\
\text { Bone } \\
\text { Ovary } \\
\text { Bladder } \\
\text { Uterus }\end{array}$ & $\begin{array}{r}37 \\
8 \\
6 \\
5 \\
5 \\
2 \\
1\end{array}$ & $\begin{array}{r}66.1 \\
14.3 \\
10.7 \\
8.9 \\
8.9 \\
3.6 \\
1.8\end{array}$ \\
\hline
\end{tabular}

Source: Patient medical records, INOR.

Table 2: Tumor Response and Survival in Patients with Inoperable Metastatic Colorectal Cancer Treated with FOLFOX-4, INOR, Havana (2001 to 2004)

\begin{tabular}{|c|c|c|}
\hline \multirow{2}{*}{ Objective Response } & \multicolumn{2}{|c|}{ Patients } \\
\hline & No. & $\%$ \\
\hline Complete Response & 7 & 12.5 \\
\hline Partial Response & 18 & 32.1 \\
\hline Stable Disease & 13 & 23.2 \\
\hline Progression & 18 & 32.1 \\
\hline Objective Response Rate & \multicolumn{2}{|c|}{$44.6 \%$} \\
\hline Median Response Duration & \multicolumn{2}{|c|}{9.6 months } \\
\hline Progression-free Survival & \multicolumn{2}{|c|}{$\begin{array}{c}\text { Median } 8.9 \text { months }(95 \% \text { Cl: } 7.5-10.2) \\
\text { 1-year rate: } 28 \% \quad 2 \text {-year rate: } 7 \%\end{array}$} \\
\hline Overall Survival & \multicolumn{2}{|c|}{$\begin{array}{cl}\text { Median } 16.9 \text { months } & (95 \% \mathrm{Cl}: 13.9-19.8) \\
1 \text {-year rate: } 75 \% & 2 \text {-year rate: } 17 \%\end{array}$} \\
\hline
\end{tabular}

$(48 \%)$ rather than the rectum $(35 \%)(p<0.92)$. The metastatic site with best response was the liver (48\% RR; 6 CR cases). Response was higher in patients who had received previous chemotherapy with regimens that included 5-FU compared to those who had not $(50 \%$ and $41 \%$ RR, respectively) $(p<0.96)$ and was also higher among patients who had undergone previous curative surgery, compared to those who had not $(58 \%$ and $22 \% \mathrm{RR}$, respectively). Among the 45 patients that completed $\geq 6$ cycles, 22 patients attained CR or PR for a $49 \%$ RR, while only 3 of the 11 patients who received $<6$ cycles responded to treatment, for a $27 \% \mathrm{RR}$. The metastases that best responded to the FOLFOX-4 regimen were hepatic (44\% RR) and pulmonary $(33 \% \mathrm{RR})$.
Median progression-free survival (PFS) for the group was 8.9 months (95\% Cl: $7.5-10.2$ months). The 1-year PFS rate was estimated at $28 \%(95 \% \mathrm{Cl}: 16.7-40.4)$ and the 2-year PFS rate at 7\% (95\% Cl: 1.46-16.39) (Table 2) (Figure 1).

Median overall survival (OS) was 16.9 months (range 13.9-19.8 months) (Cl 95\%). The 1-year OS rate was estimated at $75 \%$ (Cl 95\%: 65.7-87.8), and the 2-year OS rate at $17 \%(\mathrm{Cl} 95 \%$ : 6.89-26.8) (Table 2) (Figure 2).

Table 3 compares the main efficacy results for FOLFOX-4 in the current study with those of similar studies reported in the international literature.

Safety profile During the study, 66 grade $3(23.3 \%)$ and 9 grade $4(3.1 \%)$ adverse events were reported. The most frequent hema-

Figure 1: Cumulative 4-Year Progression-Free Survival Rate, Metastatic Colorectal Cancer Patients Treated with FOLFOX-4, INOR, Havana (2001 to 2004)

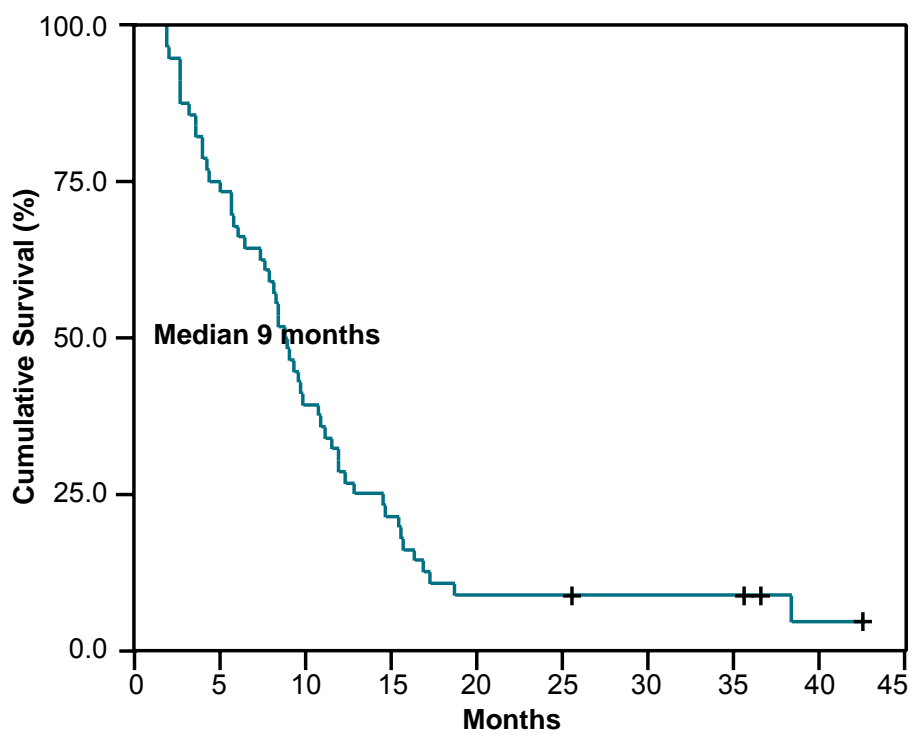

Figure 2: Cumulative 4-Year Overall Survival Rate, Metastatic Colorectal Cancer Patients Treated with FOLFOX-4, INOR, Havana (2001 to 2004)

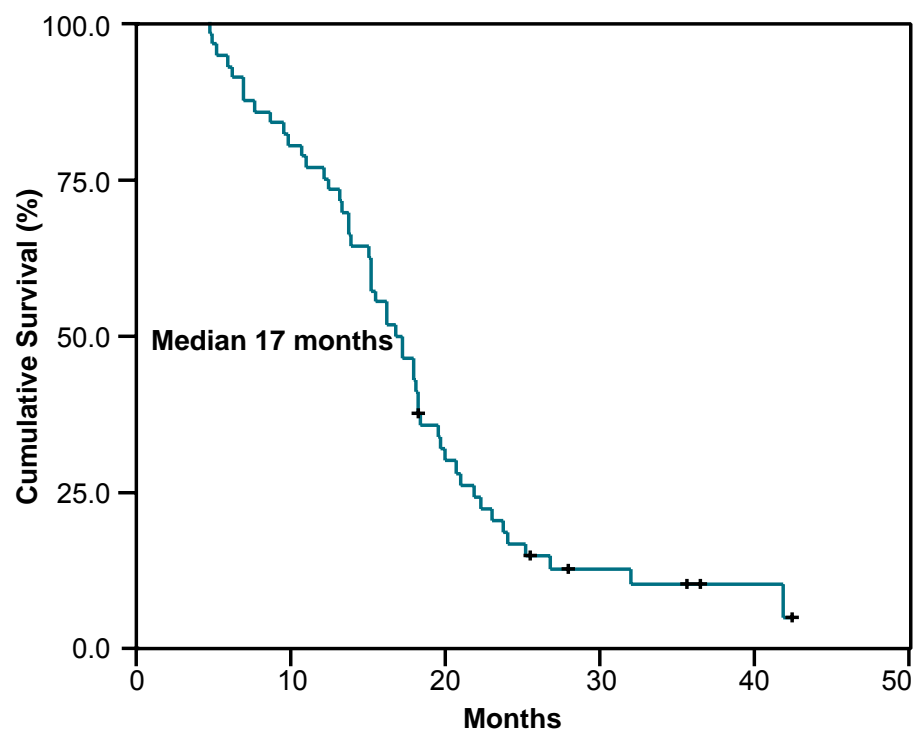


Table 3: Comparison of Tumor Response and Survival in Six Studies of FOLFOX-4 Treatment of Advanced Colorectal Cancer

\begin{tabular}{|l|r|r|r|r|}
\hline Author & No. cases & RR (\%) & $\begin{array}{c}\text { PFS } \\
\text { (months) }\end{array}$ & $\begin{array}{c}\text { OS } \\
\text { (months) }\end{array}$ \\
\hline Levi (1994)[11] & 93 & 58 & 10.0 & 15.0 \\
\hline Giacchetti (2000)[12] & 100 & 34 & 8.7 & 19.4 \\
\hline De Gramont (2000)[13] & 210 & 50 & 9.0 & 16.2 \\
\hline Tournigand (2004)[14] & 111 & 54 & 8.0 & 20.6 \\
\hline Goldberg (2004)[15] & 267 & 45 & 9.3 & 19.5 \\
\hline Lami (2009) & 56 & 45 & 8.9 & 16.9 \\
\hline
\end{tabular}

RR: Response Rate, PFS: Progression-free Survival, OS: Overall Survival

Table 4: Adverse Events Associated with FOLFOX-4 in Patients with Inoperable Metastatic Colorectal Cancer, INOR, Havana

(2001 to 2004)

\begin{tabular}{|c|c|c|c|c|}
\hline Toxicity & \multicolumn{2}{|c|}{ No. of Events } & \multicolumn{2}{|c|}{$\%$} \\
\hline Grade 1 & & & 5.7 \\
\hline Grade 2 & 192 & & & 67.8 \\
\hline Grade 3 & \multicolumn{2}{|r|}{66} & & 23.3 \\
\hline Grade 4 & \multicolumn{2}{|r|}{9} & & 3.2 \\
\hline & \multicolumn{4}{|c|}{ Patients } \\
\hline & \multicolumn{2}{|c|}{$\mathbf{N}^{\circ}$} & \multicolumn{2}{|c|}{$\%$} \\
\hline Dose Reduction & \multicolumn{2}{|r|}{13} & \multicolumn{2}{|r|}{23.2} \\
\hline Suspension of Treatment & \multicolumn{2}{|r|}{6} & \multicolumn{2}{|r|}{10.7} \\
\hline \multirow{3}{*}{ Adverse Event } & \multirow{2}{*}{\multicolumn{2}{|c|}{$\begin{array}{c}\text { General } \\
\text { (Grade 1, 2,3,4) } \\
\text { Patients }\end{array}$}} & \multicolumn{2}{|c|}{ Grade 3-4 } \\
\hline & & & \multicolumn{2}{|c|}{ Patients } \\
\hline & \begin{tabular}{l|l|}
$N^{\circ}$ \\
\end{tabular} & $\%$ & \multicolumn{2}{|c|}{\begin{tabular}{l|l}
$\mathrm{v}^{\circ}$ & $\%$ \\
\end{tabular}} \\
\hline Nausea-Vomiting & 27 & 48.2 & 3 & 5.4 \\
\hline Diarrhea & 21 & 37.5 & 2 & 3.6 \\
\hline Neutropenia & 18 & 32.1 & 4 & 7.1 \\
\hline Sensory Neurotoxicity & 15 & 26.8 & 6 & 10.7 \\
\hline Anemia & 13 & 23.2 & 1 & 1.8 \\
\hline Oral Mucositis & 10 & 17.9 & 1 & 1.8 \\
\hline Fatigue & 9 & 16.1 & 3 & 5.4 \\
\hline Abnormal Transaminase Va & 6 & 10.7 & 1 & 1.8 \\
\hline
\end{tabular}

Source: Patient medical records, INOR

tologic toxicity was neutropenia, observed in 18 patients $(32.1 \%)$ and classified grade $3-4$ in 4 cases $(7.1 \%)$. Febrile neutropenia was reported in 3 patients (5.4\%). Nausea and vomiting were reported in 27 patients (48.2\%) and classified grade $3-4$ in 3 cases (5.4\%) (Table 4).

Neurotoxicity related to oxaliplatin, characterized by peripheral sensory neuropathy, was observed in 15 patients $(26.8 \%)$ and classified as grade 3 in 6 cases (10.7\%). Palmar-plantar erythrodysesthesia (hand-foot syndrome), associated with 5-FU treatments,[10] occurred in 3 patients $(5.4 \%)$ and was classified as grade 3 in one (1.8\%). Three patients (5.4\%) exhibited hypersensitivity reactions, characterized by sweating, cutaneous dysesthesia and dyspnea, classified as grade 3 in one patient (1.8\%). Renal, cardiac and pulmonary toxicity were rarely observed and, in any case, were mild.

Dose reduction was required in 13 patients (23.2\%), primarily due to adverse events involving peripheral sensory neuropathy and severe neutropenia in previous cycles. Severe adverse reactions led to discontinuation of treatment in 6 patients $(10.7 \%)$.

\section{DISCUSSION}

This study examined the efficacy and safety profile of the FOLFOX-4 regimen as a first-line therapy for metastatic CRC. Biomedical and epidemiological characteristics of participating patients were consistent with those described in similar studies.[11-16]

Randomized clinical trials have consistently demonstrated the superiority of the FOLFOX-4 regimen compared to 5-FU-FA as a first-line therapy for metastatic CRC. In studies using the 5-FU-FA combination, a 23\% RR average, 6.1-month PFS, and 12- to14-month OS have been obtained, as reported by Levi, Giacchetti and De Gramont.[11-13] Higher response values found in our study demonstrate that better results can be obtained with the FOLFOX-4 regimen in the Cuban medical setting, where 5-FU-FA has been standard treatment until now.

A comparison of response indicators in our study, and the approximate 45\% RR, 9-month PFS and 16-month OS values described in randomized studies with similar treatment regimens,[11-15] also shows that our results fall within the expected response range. In our study, initial tumor site in the colon and a history of previous therapy-either chemotherapy based on 5-FU or surgery-were noteworthy as the strongest predictors of response to FOLFOX-4 therapy.

In the FOLFOX-4 regimen, the toxicity profile of fluoropyrimidines administered by continuous infusion (characterized by diarrhea, mucositis and hand-foot syndrome) is compounded by that of oxaliplatin, characterized by peripheral sensory neurotoxicity in $10 \%-20 \%$ of patients treated.[17,18]

During the study, $26.5 \%$ of total adverse events described were classified grade $3-4$. Neurotoxicity headed this list, followed by neutropenia, fatigue, and vomiting.

Peripheral sensory neuropathy and, to a lesser degree, neutropenia, were the main causes of dose reduction and suspension of treatment. Analysis of the adverse events described found that these were consistent with the general toxicity profile for regimens combining oxaliplatin with fluoropyrimidines, documented in international studies.[11-15]

It is worth noting that most of the patients affected by peripheral neuropathy experienced partial or complete recovery during the follow-up period, as described in studies such as MOSAIC. [19] Large FOLFOX-4 regimen trials, such as MOSAIC[19] and OPTIMOX,[20,21] report neutropenia as a toxic effect in $42 \%$ and $33 \%$ of the sample, respectively, and febrile neutropenia in $2 \%$. Our study reported neutropenia and febrile neutropenia in $32 \%$ and $5 \%$ of patients, respectively, coinciding with the mean values reported in those trials. In contrast to other studies, [22] mucositis, diarrhea and hand-foot syndrome were infrequently classified as severe adverse events in our study.

The impact of FOLFOX-4 therapy on patients with metastatic $\mathrm{CRC}$ demonstrated in this study raises the need for new therapies in combination with the FOLFOX-4 regimen or as a subsequent treatment. Among the cytotoxins shown to be highly effective against CRC is irinotecan, which, in combination with $5-\mathrm{FU}$, has proved to be similar in efficacy to regimens combining oxaliplatin with 5-FU, and which can be used after progression with the FOLFOX-4 regimen, obtaining positive responses with a median survival of up to 21 months.[23] Due to the high cost of irinotecan, however, applying it in the Cuban health system has not been possible to date. 
Another treatment option currently being tested in multiple international studies is the combination of regimens such as FOLFOX-4 with monoclonal antibodies. Examples include chemotherapy combined with bevacizumab (monoclonal antibody targeting the vascular endothelial growth factor), or cetuximab (monoclonal antibody targeting the epidermal growth factor), which can increase survival rates by 2-3 months.[24,25] The oncology department at INOR and the Molecular Immunology Center in Havana, informed by the results of this study, are currently conducting a Phase I-II clinical trial to evaluate safety of the FOLFOX-4 regimen combined with nimotuzumab (a Cuban-developed monoclonal antibody targeting the epidermal growth factor)[26] as a first-line treatment in patients with metastatic colorectal cancer.

A limitation of the present study is that only INOR patients were included. Including patients from other medical centers would have increased the number of cases analyzed and thus the impact of the study. Nevertheless, the findings are important for Cuban oncology in that they have introduced new therapeutic options for colorectal cancer. As a result of this and other studies, the FOLFOX-4 regimen is now considered one of the standard treatments for metastatic colorectal cancer in Cuba, and studies are underway to evaluate its application as an adjuvant treatment.

\section{CONCLUSIONS}

Given the response rates attained and the toxicity profile observed in this study, we can conclude that the FOLFOX-4 regimen is an effective and well-tolerated therapeutic option for treatment of inoperable metastatic colorectal cancer in Cuba, and is therefore recommended as a first-line treatment for these patients in the Cuban health care system. Further studies of FOLFOX-4 should be carried out in combination with new therapeutic agents developed by Cuban biotechnology, in the search for greater efficacy. -1 -

\section{REFERENCES}

1. Jemal A, Murray T, Ward E, Murray T, Xu J, Smigal $C$, et al. Cancer statistics, 2006. CA Cancer J Clin. 2006 Mar-Apr;56(2):106-30

2. DeVita VT, Hellman S, Rosenberg SA. Colon cancer. In: Pine J, Murphy J, Sebring S, editors. Cancer: Principles and Practice of Oncology. New York: Lippincott Williams \& Wilkins;2005.

3. Anuario Estadístico de Salud 2007. Havana: Ministry of Public Health, National Statistics Division; 2007 [updated 2008 August 5; cited 2009 Jun 5]. Available from: http://www.sld.cu/ servicios/estadisticas/.

4. Meyerhardt JA, Mayer RJ. Systemic Therapy for Colorectal Cancer. N Engl J Med. 2005 Feb 3;352(5):476-87.

5. Raymond E, Buquet-Fagot C, Djelloul S, Mester $\mathrm{J}$, Cvitkovic E, Allain P, et al. Antitumor activity of oxaliplatin in combination with 5-fluorouracil and the thymidylate synthase inhibitor AG337 in human colon, breast and ovarian cancers. Anticancer Drugs. 1997 Oct:8(9):876-85.

6. Scheithauer W, Rosen H, Kornek GV, Sebesta C, Depisch D. Randomised comparison of combination chemotherapy plus supportive care with supportive care alone in patients with metastatic colorectal cancer. BMJ. 1993 Mar 20;306(6880):752-5.

7. Benites CM. Cáncer Colorrectal. Supervivencia [thesis]. Havana (CU): National Institute of Oncology and Radiobiology;1986.

8. Cella DF, Tulsky DS, Gray G, Sarafian B, Linn E, Bonomi A, et al. The Functional Assessment Cancer Therapy Scale: development validation of the general measure. J Clin Oncol. 1993 Mar;11(3):570-9.

9. Therasse P, Arbuck SG, Eisenhauer EA, Wanders J, Kaplan RS, Rubinstein L, et al. New guidelines to evaluate the response to treatment in solid tumors. J Natl Cancer Inst. 2000 Feb 2;92(3):205-16.

10. National Cancer Institute [Internet]. Rockville (MD): Cancer Therapy Evaluation Program, Common Terminology Criteria for Adverse Events, Version 3.0, DCTD, NCl, NIH, DHHS (US); [updated 2008 May 29; cited 2007 June 17]. Available from: http://www.ctep.cancer.gov/.

11. Levi FA, Zidani R, Vannetzel JM, Perpoint B, Focan C, Faggiuolo R, et al. Chronomodulated vs. fixed-infusion-rate delivery of ambulatory chemotherapy with oxaliplatin, fluorouracil, and folinic acid (leucovorin) in patients with colorectal cancer metastases: A random- ized multi-institutional trial. J Natl Cancer Inst. 1994;86(21):1608-17.

12. Giacchetti S, Perpoint B, Zidani R, Le Bail N, Faggiuolo R, Focan C, et al. Phase III multicenter randomized trial of oxaliplatin added to chronomodulated fluorouracil-leucovorin as first-line treatment of metastatic colorectal cancer. J Clin Oncol. 2000 Jan;18(1):136-47.

13. De Gramont A, Figer A, Seymour M, Homerin M, Hmissi A, Cassidy J, et al. Leucovorin and fluorouracil with or without oxaliplatin as first-line treatment in advanced colorectal cancer. J Clin Oncol. 2000 Jan;18(1):136-47.

14. Tournigand $\mathrm{C}$, André $\mathrm{T}$, Achille $\mathrm{E}$, Lledo $\mathrm{G}$, Flesh $M$, Mery-Mignard D, et al. FOLFIRI followed by FOLFOX- 6 or the reverse sequence in advanced colorectal cancer: a randomized GERCOR study. J Clin Oncol. 2004 Jan 15;22(2):229-37.

15. Goldberg RM, Sargent DJ, Morton RF, Fuchs CS, Ramanathan RK, Williamson SK, et al. A randomized controlled trial of fluorouracil plus leucovorin, irinotecan, and oxaliplatin combinations in patients with previously untreated metastatic colorectal cancer. J Clin Oncol. 2004 Jan 1;22(1):23-30. Epub 2003 Dec 9.

16. Sobrero A, Guglielmi A, Grossi F, Puglisi F, Aschele $\mathrm{C}$. Mechanism of action of fluoropyrimidines: relevance to the new developments in colorectal cancer chemotherapy. Semin Oncol. 2000 Oct;27(5 Suppl 10):S72-7.

17. Schmoll $\mathrm{HJ}$. The role of Oxaliplatin in the treatment of advanced metastatic colorectal cancer: prospects and future directions. Semin Oncol. 2002 Oct;29(5 Suppl 15):S34-9.

18. Grothey A. Oxaliplatin-safety profile: neurotoxicity. Semin Oncol. 2003 Aug;30(4 Suppl 15):S5-13.

19. De Gramont A, Boni C, Navarro M, Tabernero J, Hickish T, Topham C, et al. Oxaliplatin/5FU/LV in the adjuvant treatment of stage II and stage III colon cancer: efficacy results with a median follow-up of 4 years. J Clin Oncol. 2005 Jun 1;23(16 Suppl):S3501.

20. Pérez-Staub N, Lledo G, Paye F, Gayet B, Flesch $M$, Cervantes $A$, et al. Surgery of colorectal metastasis in the Optimox 1 study. J Clin Oncol. 2006 Jun 20;24(18 Suppl):S3522.

21. Maindrault-Goebel F, Lledo G, Chibaudel B, Mineur L, Andre T, Bennamoun M, et al. OPTIMOX2, a large randomized phase II study of maintenance therapy or chemotherapyfree intervals after FOLFOX in patients with metastatic colorectal cancer. A GERCOR study. J Clin Oncol. 2006 Jun 20;24(18 Suppl):S3504.

22. Wiseman LR, Adkins JC, Plosker GL, Goa KL. Oxaliplatin: a review of its use in the management of metastatic colorectal cancer. Drugs Aging. 1999 Jun;4(6):459-75

23. Omura K. Advances in chemotherapy against advanced or metastatic colorectal cancer. Digestion. 2008;77(Suppl 1):S13-22.

24. Hurwitz H, Fehrenbacher L, Novotny W, Cartwright T, Hainsworth J, Heim W, et al. Bevacizumab plus Irinotecan, Fluorouracil, and Leucovorin for Metastatic Colorectal Cancer. N Engl J Med. 2004 Jun 3;350:2335-42.

25. Moosmann N, Heinemann C. Cetuximab plus oxaliplatin-based chemotherapy in the treatment of colorectal cancer. Expert Rev Anticancer Ther. 2008 Mar;8(3):319-29.

26. Crombet T, Torres L, Nenínger E, Catalá M, Solano ME, Perera A, et al. Pharmacological evaluation of humanized anti-epidermal growth factor receptor, monoclonal antibody $\mathrm{h}-\mathrm{R} 3$, in patients with advanced epithelial-derived cancer. J Immunother. 2003 Mar-Apr;26(2):139-48.

\section{THE AUTHORS}

Leonardo Lami Casaus (Corresponding Author: leo.lami@infomed.sld.cu), oncologist, National Oncology and Radiobiology Institute, Havana, Cuba.

Fernando Areces Delgado, oncologist, National Oncology and Radiobiology Institute, Havana, Cuba.

Juan J Lence Anta, biostatistician, National Oncology and Radiobiology Institute, Havana, Cuba.

María A Arbesú Michelena, master of pharmaceutical science, National Oncology and Radiobiology Institute, Havana, Cuba.

Submitted: April 28, 2008

Approved for publication: June 4, 2009 